\title{
Quelles variétés de sorgho pour satisfaire les exigences des productrices de malt et de dolo au Burkina Faso?
}

\author{
Laurencia T. SONGRE-OUATTARA ${ }^{1}$, Roger KABORE 2 , Jonas M. KAM ${ }^{3}$, Charlotte KONKOBO- \\ YAMEOGO ${ }^{1}$, Joachim OUEDRAOGO ${ }^{4}$, Gilles TROUCHE 5
}

1 Institut de Recherche en Sciences Appliquées et Technologies (IRSAT), Département Technologie Alimentaire (DTA), 03 BP 7047 Ouagadougou 03, Burkina Faso,

${ }^{2}$ Association Minim Song Panga (AMSP), BP 268, Kaya, Burkina Faso,

${ }^{3}$ Institut National des Sciences des Sociétés (INSS), Burkina Faso

${ }^{4}$ Fédération nationale des Industries de l'Agroalimentaire et de transformation du Burkina (FIAB), 01 BP 6178 Ouaga 01, Burkina Faso

${ }^{5}$ CIRAD, UMR AGAP, F-34398 Montpellier, France

Auteur correspondant : Email : laurenciaouattara@yahoo.fr Tél : 0022670278633

Original submitted in on $22^{\text {nd }}$ July 2016. Published online at www.m.elewa.org on $31^{\text {st }}$ October 2016

http://dx.doi.org/10.4314/jab.v106i1.9

\section{RÉSUMÉ}

Objectif : L'objectif de cette étude était d'identifier les types et caractéristiques de grains préférés par les transformatrices pour produire une bonne qualité de malt de sorgho et/ou la bière traditionnelle de sorgho communément appelée dolo.

Méthodologie et résultats: Des enquêtes semi-structurées ont été réalisées en 2015 dans dix localités réparties dans trois provinces du Burkina Faso (Kadiogo, Kouritenga, Mouhoun) auprès d'un échantillon global de 105 malteuses et de 105 brasseuses de dolo choisies de manière aléatoire. L'analyse des données d'enquêtes a été réalisée avec le logiciel Sphinx. Les résultats ont montré que le sorgho rouge est le type de grain préféré pour le maltage et le brassage du dolo. Les malteuses préfèrent le sorgho rouge surtout pour son aptitude à bien germer. Au sein des variétés de sorgho rouge, les grains durs sont les plus appréciés des brasseuses. Cependant, il existe des différences régionales dans ces préférences. Dans le Kadiogo, les brasseuses préfèrent autant les grains rouges et légers que les grains rouges et lourds tandis que dans le Kouritenga, les préférences vont en premier lieu pour les grains rouges lourds et durs puis pour les grains rouges et lourds. Quant au Mouhoun, les types de grains préférés par les brasseuses sont plus diversifiés, avec une légère préférence pour les grains rouges, lourds et durs, et les grains blancs et lourds sont assez bien appréciés.

Conclusion et application des résultats : Cette étude a permis d'identifier les types et caractéristiques de grain de sorgho désirés par les transformatrices de malt et de dolo. Elle fournit ainsi des informations techniques aux sélectionneurs pour la création des variétés de sorgho destinées à la production artisanale de malt et de bière de sorgho.

Mots clés : Sorgho, qualité de grain, malt, bière traditionnelle 


\section{What varieties of sorghum meet the demands of producers of malt and dolo in Burkina Faso ABSTRACT}

Objective: The objective of this study was to identify the types and characteristics of grain preferred by the processors to produce a good quality of sorghum malt and/or traditional sorghum beer commonly known as dolo.

Methodology and results: Semi-structured surveys were conducted in 2015 in 10 locations in three provinces of Burkina Faso (Kadiogo, Kouritenga, Mouhoun) from a sample of 105 brewers and 105 dolo producers selected randomly. The data of the survey were analysed using Sphinx software. The results showed that red sorghum is the type of grain preferred for malting and brewing of the dolo. The brewers prefer red sorghum mainly for its ability to germinate well. Within the varieties of red sorghum, hard grains are the most popular among the brewers. However, there are regional differences in those preferences. In the Kadiogo, the brewers prefer both red and light grains and red and heavy grains while in the Kouritenga, preferences are primarily for red, heavy and hard grain, and then for red and heavy grains. As for the Mouhoun, types of grain preferred by brewers are more diverse, with a slight preference for red, heavy and hard grains, and, white and heavy grains are fairly appreciated.

Conclusion and implementation of the results: This study identified the types and characteristics of sorghum grains desired by the malt and dolo processors. It thus provides technical information to breeders for the creating sorghum varieties for of the artisanal production of malt and dolo.

Keywords: sorghum, malt, grain quality, traditional beer

\section{INTRODUCTION}

$\mathrm{Au}$ Burkina Faso, la production céréalière est caractérisée par une prédominance du sorgho dont la production annuelle est estimée à 1707 613 tonnes, soit $38,20 \%$ de la production céréalière totale (MARHASA/DGESS, 2015). Sur l'ensemble de la production du sorgho, la part du sorgho blanc est de $75 \%$ contre $25 \%$ pour le sorgho rouge. Dans plusieurs pays d'Afrique, le grain de sorgho est la principale céréale utilisée dans la production des bières traditionnelles (Taylor et Dewar, 2001; Thaoge et al., 2003; Traoré et al., 2004 ; Palé et al., 2010 ; Maha et al., 2014 ). Au Burkina Faso, plus de 30\% de la production totale de sorgho, sont maltés chaque année principalement pour la fabrication d'une bière traditionnelle alcoolisée, communément appelée «dolo» (DSA-BF, 2001) dont la production est estimée à 36 millions de litres par an (Hounhouigan et Fliedel, 2010). Ce dolo couramment consommé à travers le Burkina Faso, joue un rôle culturel et socioéconomique important. En effet, il connait une grande utilisation lors des cérémonies familiales et traditionnelles, festives et funéraires, et constitue une source importante de revenus pour les productrices (Belliard, 2001). Aussi, il est d'un prix plus accessible que les bières industrielles. Le procédé traditionnel de préparation du dolo implique principalement trois phases, le maltage des grains pour l'obtention du malt, le brassage et la fermentation, pour lesquelles peuvent apparaître des variations suivant les pratiques et les matières premières utilisées (Broutin et al., 2003; Palé et al., 2010; Lyumugabe et al., 2012). A l'instar des autres bières traditionnelles d'Afrique de l'Ouest, comme le tchakpalo de la Côte d'Ivoire, le pito du Ghana, le tchoukoutou du Bénin, le dolo est une bière opaque, en raison de particules d'amidon et de levure, de couleur foncée (brun-rosâtre) dû à la présence de certains pigments provenant du malt, souvent aigre ou acide avec un $\mathrm{pH}$ de 4-5, nutritive car riche en calorie et vitamines et faiblement alcoolisée de 2 à $4 \%$ (Chevassus-Agnes et al., 1979; Broutin et al., 2003; Kayodé et al., 2005; Dicko et al., 2006). Cependant, les caractéristiques du malt de sorgho et du dolo sont variables, du fait de la diversité des variétés et des procédés utilisés (Dicko et al., 2006; Tchuenbou, 2006 ; Palé et al., 2010 ; Lyumugabe et al., 2012 ; Christian Konfo et al., 2014) qui, eux aussi varient selon les acteurs, les ethnies et la localisation géographique (Haggblade et Holzapfel, 2004 ; Tchuenbou, 
2006). Par exemple au Burkina Faso, le dolo connait différentes appellations en fonction de l'ethnie : il y'a le dolo mossi, le dolo dagara, le dolo samo, le dolo gourmantché qui varient dans leur texture, leur contenu nutritionnel, leur degré en alcool et leur propriété sensorielle. Cette variabilité est influencée par de nombreux facteurs intrinsèques et extrinsèques notamment d'ordre physique, biochimique et technologique à toutes les étapes du processus qui affectent considérablement la qualité des produits intermédiaires et du produit final. II existe une grande diversité de variétés de sorgho pour les caractères de grains, en particulier pour la couleur, la structure de l'endosperme, la dureté, la taille, le poids d'un grain, la pigmentation et les caractéristiques biochimiques (Rai et al., 2008 ;

\section{MATERIELS ET METHODES}

Zones de l'étude : L'étude a été conduite dans trois provinces du Burkina Faso que sont: Kadiogo, Mouhoun et Kouritenga (Figure 1). Ces provinces ont été sélectionnées de manière consensuelle lors d'un atelier d'échanges multi-acteurs sur les utilisations actuelles et potentielles du sorgho en milieux urbains et périurbains au Burkina Faso. Les critères de sélection étaient la forte activité de production et de commercialisation et/ou de consommation du sorgho. Dans chaque province, l'étude a concerné les chef-lieu de province plus deux (02) quartiers et ses périphéries ou deux (02) villages situés dans un rayon de $30 \mathrm{~km}$ des chefs-lieux, touchant ainsi un total de dix (10) localités. La province du Kadiogo est située dans la région du Centre. L'étude a été conduite à
Malamo et al., 2013 ; Saleh et al., 2013 ; SongréOuattara et al., 2015). Pour la brasserie, la qualité d'un malt est définie par son pouvoir diastasique et son extrait sec soluble. Ces paramètres de qualité sont déterminés principalement par la dureté et le poids des grains (Wenzel et Pretorius, 1994; Tchuenbou, 2006). Cependant, très peu informations existent sur les caractéristiques des grains préférés par les malteuses et les brasseuses pour une production de malt et de dolo de bonne qualité. L'objectif de cette étude était de déterminer les besoins des malteuses et des brasseuses de dolo sur les types et caractéristiques des grains de sorgho en vue de produire du malt et du dolo de bonne qualité selon les provinces du Burkina Faso.

Ouagadougou, le chef-lieu de province, précisément dans deux quartiers de la ville, Pissy (partie Ouest) et la Zone 1 (partie Est), plus un de leurs quartiers périphériques, respectivement Sondogo et Taabtenga. La province du Mouhoun est située au nord-ouest, dans la région de la Boucle du Mouhoun. Dans cette province, l'étude a été conduite à Dédougou, chef-lieu de la province, et dans les villages de Soukuy et Wetina situées respectivement à 15 et $23 \mathrm{~km}$ de Dédougou. La province du Kouritenga est située au Centre-Est. L'étude a été conduite dans cette province à Koupéla, chef-lieu de province, et dans les villages de Songretenga et Andemtenga, situées respectivement à 15 et $18 \mathrm{~km}$ de Koupéla. 


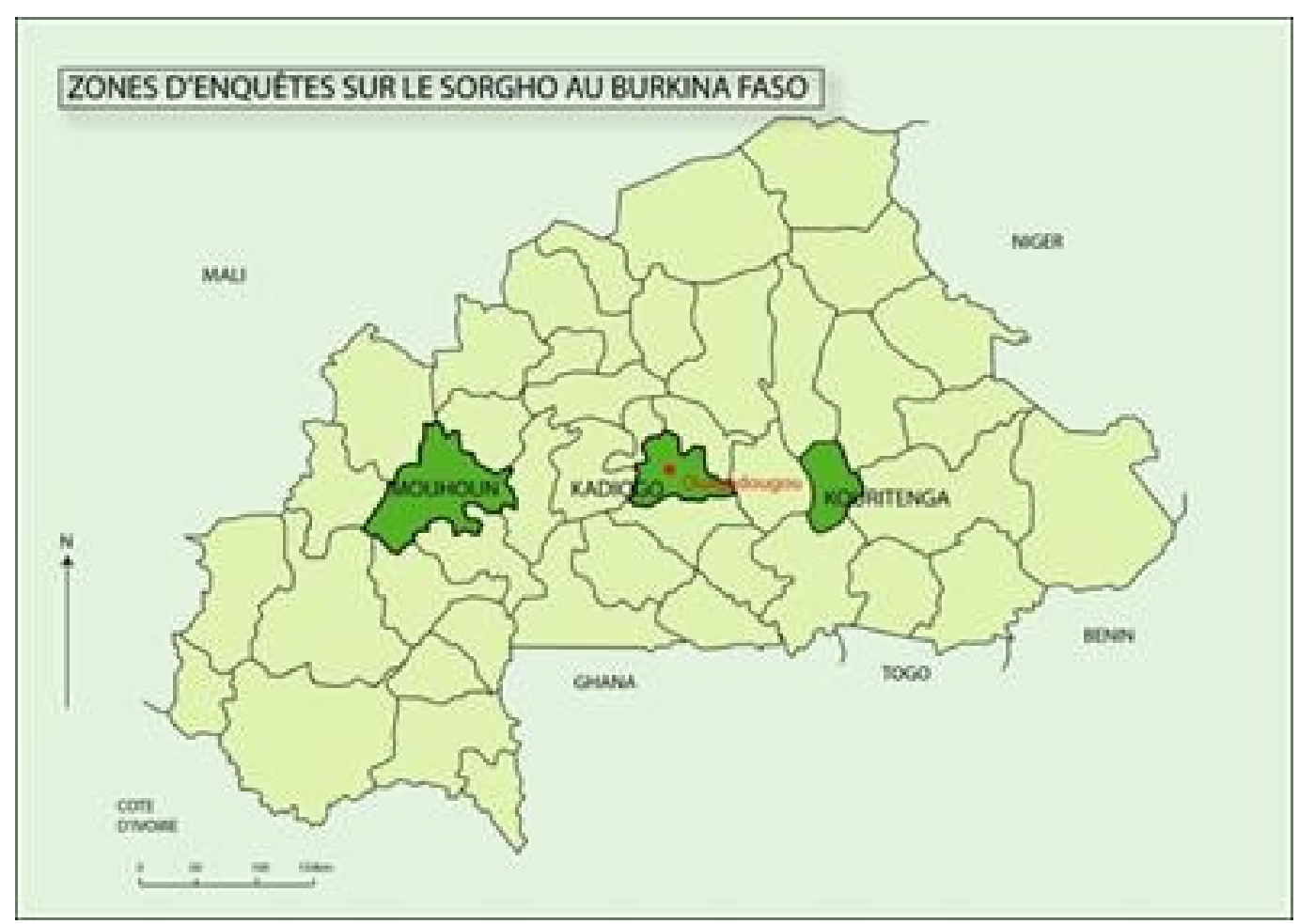

Figure 1 : Localisation des trois provinces considérées dans cette étude

Collecte des données d'enquêtes : Des enquêtes semi-structurées ont été réalisées auprès des malteuses et des brasseuses opérant dans les localités des provinces sélectionnées. Le but poursuivi était de collecter des informations sur les activités de maltage du sorgho et de brassage du dolo, et d'identifier les caractéristiques des grains de sorgho appropriés pour le malt et le dolo de sorgho. Ces entretiens étaient articulés autour des points suivants: les pratiques de maltage et qualité du malt, maltage et variétés de sorgho utilisées, qualité de malt et type de grains de la variété de sorgho, critères de qualité pour le choix du malt et ou des grains ou variétés de sorgho. La collecte des données de terrain a été effectuée entre mai et juin 2015 dans chaque province. Au total 105 malteuses et 105 brasseuses, soit 35 par province ont été enquêtées (Tableau 1). Dans toutes les provinces, ces deux catégories d'acteurs étaient sélectionnées de façon aléatoire, soit 15 dans la ville et 10 par village enquêtée pour les provinces du Mouhoun et du Kouritenga, tandis que dans la province du Kadiogo, la taille de l'échantillon variait entre 7 et 10 par localité.

Tableau 1 : Répartition des enquêtés selon la province et la localité

\begin{tabular}{l|l|l|l}
\hline Provinces & Localités & $\begin{array}{l}\text { Effectif de malteuses } \\
\text { enquêtées }\end{array}$ & $\begin{array}{l}\text { Effectif de brasseuses-dolotières } \\
\text { enquêtées }\end{array}$ \\
\hline Kadiogo & Zone 1 & 9 & 9 \\
& Pissy & 10 & 9 \\
& Sondogo & 9 & 7 \\
& Taabtenga & 7 & 10 \\
\hline Mouhoun & Dédougou & 15 & 15 \\
& Wétina & 10 & 10 \\
& Soukuy & 10 & 10 \\
\hline Kouritenga & Koupèla & 15 & 15 \\
& Songretenga & 10 & 10 \\
& Andemtenga & 10 & 10 \\
\hline Total & 105 & 105 \\
\hline
\end{tabular}




\section{Songre-Ouattara et al. J. Appl. Biosci. 2016 Quelles variétés de sorgho pour satisfaire les exigences}

des productrices de malt et de dolo au Burkina Faso?

Traitement et analyse des données : Le traitement des données s'est effectué en trois phases: la saisie des questionnaires, la codification des questions et le tri à plat. L'analyse des données d'enquêtes a été

\section{RESULTATS}

Caractéristiques de grains recherchées pour la production du malt de sorgho

Pratiques de maltage : Les résultats de notre enquête ont montré que plus de $72,5 \%$ des malteuses enquêtées ont plus de 10 ans d'expérience. Les fréquences de production du malt de sorgho sont de 1 à 3 fois par semaine, une fois par quinzaine et 1 fois par mois. Toutefois, le malt est produit majoritairement deux fois par semaine par la moitié des malteuses $(50,5 \%)$. Pour l'ensemble des malteuses enquêtées, la durée du processus de maltage varie de 3 à 10 jours avec une moyenne de $6,5 \pm 1,7$ jours. Au niveau provincial, la durée du maltage est plus longue au Kouritenga avec une moyenne de $8 \pm 1$ jours, tandis qu'elles sont plus courtes dans les provinces du Kadiogo et du Mouhoun, respectivement de 6,3 \pm 0,9 et $4,9 \pm 1,1$ jours.

Maltage et variétés de sorgho : Pour les malteuses, deux types de variétés de sorgho sont utilisés dans la production du malt: les variétés à grain rouge et les réalisée avec le logiciel Sphinx. Le test de Khi-2 a été utilisé pour tester l'indépendance entre les variables de l'étude.

variétés à grain blanc, qui seront respectivement désignées sorgho rouge et sorgho blanc. Plus de 2/3 des malteuses utilisent le sorgho rouge, soit $72,5 \%$ contre $27,5 \%$ pour le sorgho blanc. Pour les utilisatrices de sorgho rouge, les principales raisons qui expliquent leur choix sont la qualité des produits de malt et de dolo, l'appréciation par les clients et la disponibilité sur le marché, respectivement par $39 \%, 23 \%$ et $12,5 \%$ des malteuses. Pour le sorgho blanc, son utilisation est faite à défaut, notamment en cas de non disponibilité du sorgho rouge.

Critères d'un malt de bonne qualité : De nombreux critères sont utilisés par les malteuses dans l'appréciation d'un malt de bonne qualité. Parmi ces critères, les quatre plus importants sont: le taux de germination $(24,5 \%)$, le goût sucré $(22,4 \%)$, l'odeur $(21,6 \%)$ et la couleur $(14,5 \%)$. La longueur des germes ou radicule, l'intégrité du grain après germination et la propreté du malt apparaissent au deuxième plan dans l'appréciation d'un malt de bonne qualité (Figure 2).

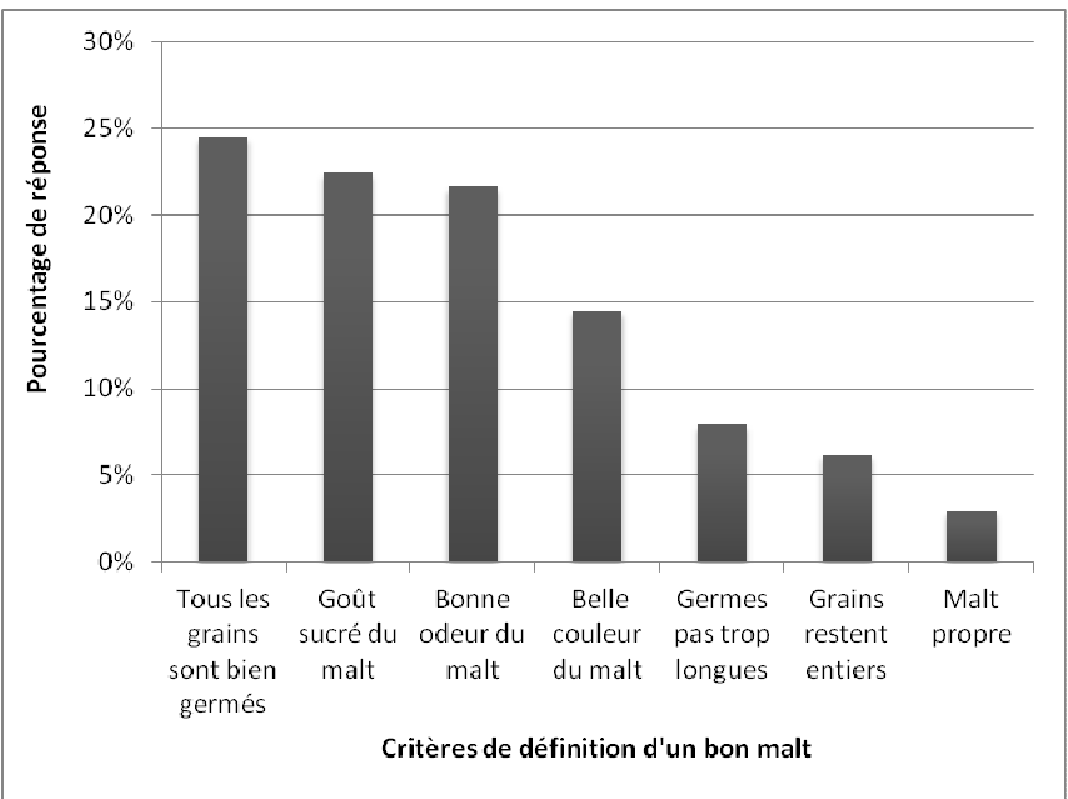

Figure 2 : Critères définissant un malt de bonne qualité

La Figure 3 présente les critères définissant un malt de bonne qualité par les malteuses de chaque province. Dans la province du Kadiogo, les principaux critères de qualité d'un bon malt pour les malteuses sont dans l'ordre décroissant, le goût sucré, la germination de tous les grains et l'odeur du malt. Pour les malteuses 


\section{Songre-Ouattara et al. J. Appl. Biosci. $2016 \quad$ Quelles variétés de sorgho pour satisfaire les exigences des productrices de malt et de dolo au Burkina Faso?}

du Mouhoun, la bonne germination, l'odeur du malt et l'intégrité des grains sont les principaux déterminants tandis que dans le Kouritenga, le goût sucré, la couleur et l'odeur du malt sont les premiers déterminants de la qualité. La bonne germination et l'odeur du malt sont donc les deux critères importants communs à l'ensemble des malteuses enquêtées tandis que le goût sucré apparait comme le premier critère dans les provinces du Kadiogo et du Kouritenga.

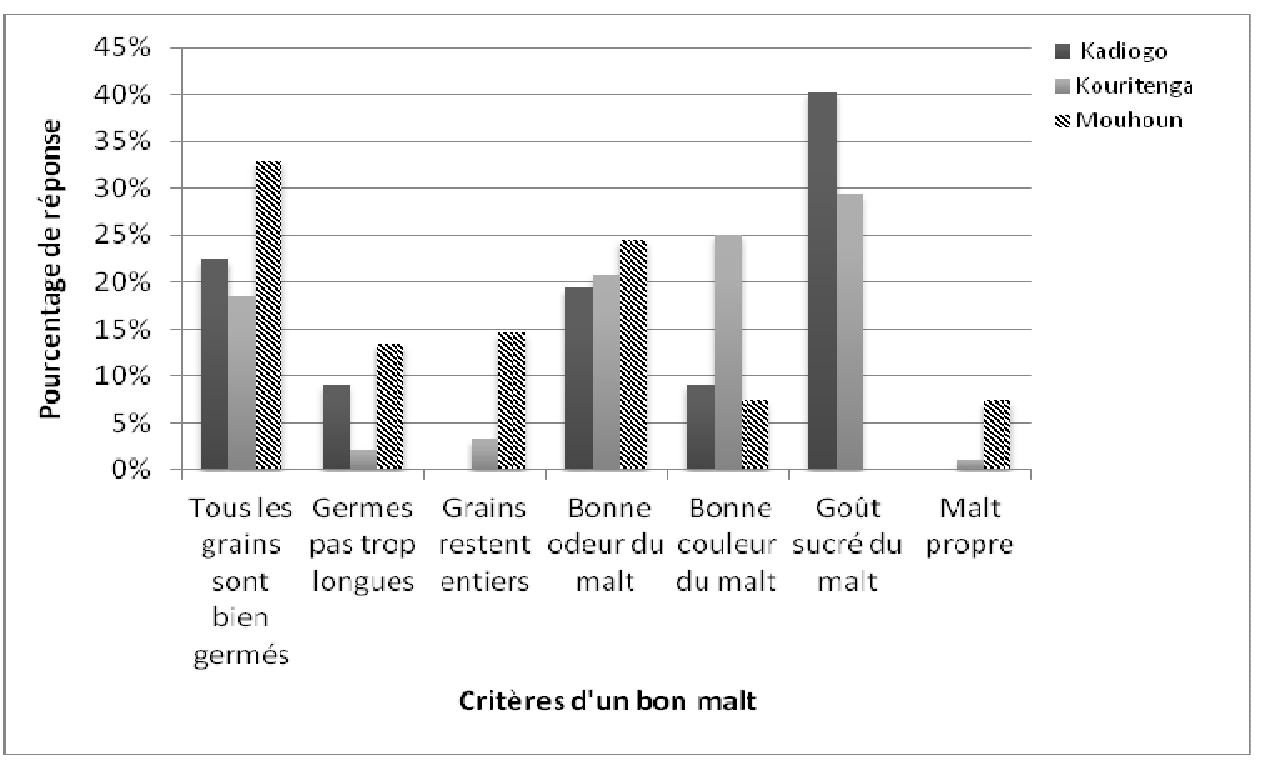

Figure 3 : Définition des critères d'un malt de bonne qualité selon la province

Caractéristiques des grains des variétés de sorgho utilisés par les malteuses : Les enquêtes révèlent que toutes les malteuses enquêtées utilisent préférentiellement les grains de sorgho rouge, car cela convient mieux à leur procédé de transformation et/ou à la qualité finale désirée du malt et cela sur la base de leur expérience. Les grains achetés chez les commerçants sont en règle générale des mélanges de plusieurs variétés ce qui ne permet pas aux malteuses de bien connaitre les variétés qu'elles utilisent et donc de préciser leurs noms. Certaines malteuses, surtout celles des villages, achètent directement les variétés de caractéristiques désirées auprès de certains producteurs, dans les marchés villageois; dans ce cas ces malteuses sont généralement capables d'identifier les variétés utilisées. $100 \%$ des malteuses enquêtées, utilisent le sorgho rouge pour le maltage du fait de sa rapidité de germination et de la longue conservation du malt. Les caractéristiques de grains durs, grains lourds et gros grains sont par ordre d'importance les descripteurs principaux des variétés de sorgho rouge utilisées. Pour les grains de sorgho blanc, le caractère dur du grain reste le plus recherché.
Demandes de nouvelles variétés : La majorité des malteuses enquêtées, soit $60 \%$ sont favorables à utiliser de nouvelles variétés pour produire le malt. II existe toutefois une dépendance très significative entre le taux d'acceptation de nouvelles variétés de sorgho et la province considérée (1- $p \geq 99,99 \%)$. Ainsi, dans les provinces du Mouhoun et du Kadiogo, respectivement $94 \%$ et $51 \%$ des malteuses sont favorables à l'utilisation d'une nouvelle variété de sorgho tandis que dans le Kouritenga $66 \%$ des malteuses y sont réticentes. Sur l'ensemble des 10 localités, les trois caractéristiques de qualité de grain les plus désirées pour les nouvelles variétés de sorgho sont la bonne germination $(42 \%)$, la couleur rouge $(22 \%)$ et la dureté du grain (17\%). Les autres critères moins déterminants cités par les malteuses sont la lourdeur du grain, l'odeur, le goût sucré, la couleur blanche, la légèreté et la grosseur du grain (Figure 4A). Le classement de ces critères varie peu entre les trois provinces. Toutefois dans la province du Kadiogo les malteuses ont exprimé un plus faible nombre de critères de choix mais sont les seules demandeuses de grains de couleur blanche (Figure 4B). 


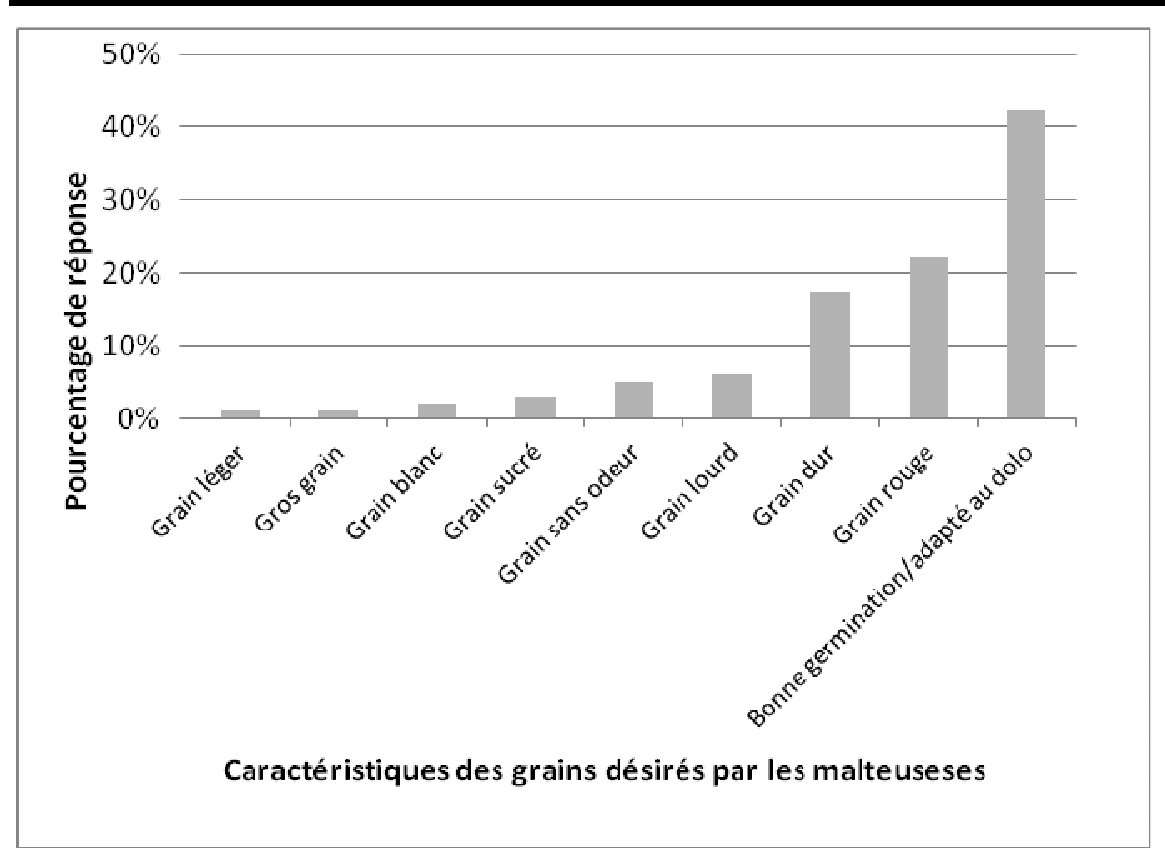

Figure 4A : Caractéristiques de grains désirées par les malteuses pour les nouvelles variétés de sorgho pour l'ensemble des 10 localités étudiées

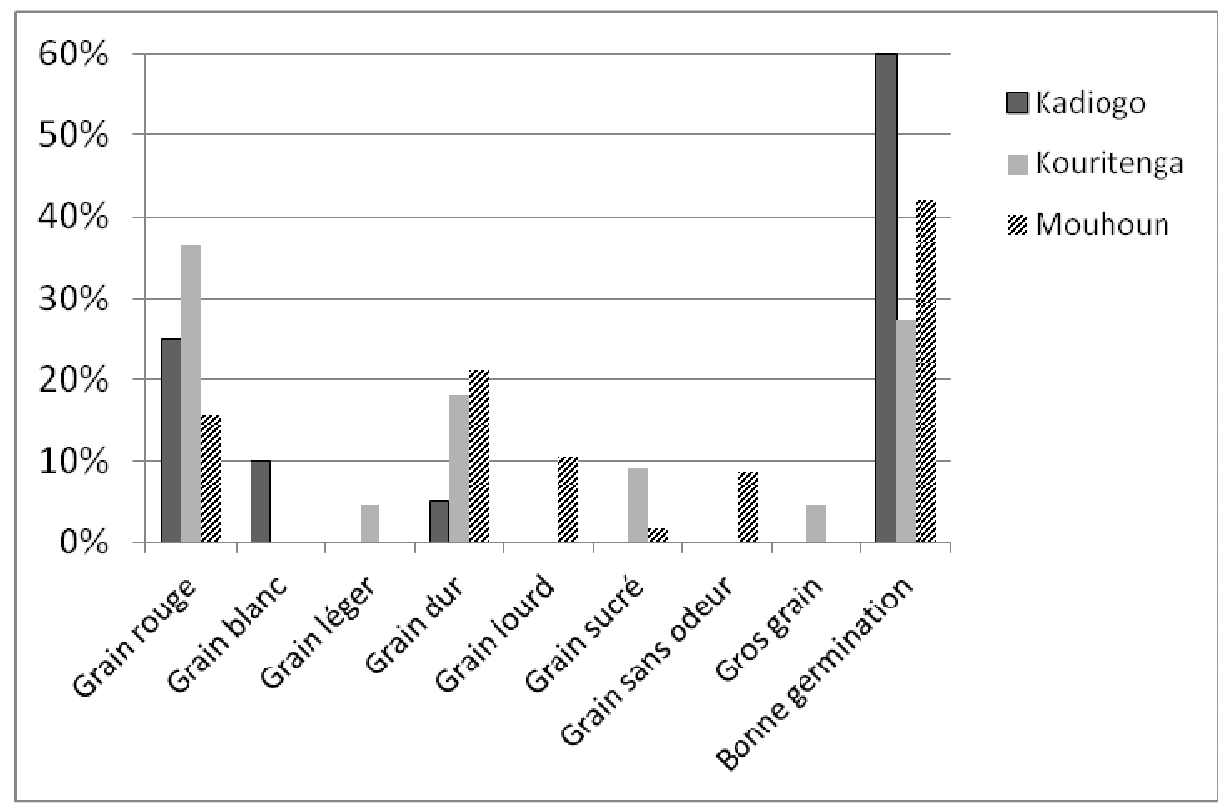

Figure 4B : Caractéristiques de grains désirées par les malteuses pour les nouvelles variétés de sorgho selon chaque province

Caractéristiques de grains recherchées pour la fabrication du dolo : Parmi les 105 brasseuses de dolo interviewées dans cette étude, $51,4 \%$ exercent également l'activité de maltage mais cette proportion varie considérablement en fonction de la province d'origine. Ainsi, $100 \%$ des brasseuses du Mouhoun sont aussi malteuses alors que cette catégorie est seulement de 28,6\% au Kouritenga et $23,50 \%$ au Kadiogo.

Critères de qualité d'un bon dolo : Pour l'ensemble des brasseuses enquêtées, les principaux critères qui définissent un dolo de bonne qualité sont le goût du dolo qui doit être sucré et surtout pas aigre pour $27 \%$, la couleur rouge de préférence pour $20 \%$ et le taux 


\section{Songre-Ouattara et al. J. Appl. Biosci. $2016 \quad$ Quelles variétés de sorgho pour satisfaire les exigences des productrices de malt et de dolo au Burkina Faso?}

élevé d'alcool pour $17 \%$. Au niveau provincial, ces critères de qualité apparaissent dans un ordre différent. En effet, dans le Mouhoun, le taux d'alcool, la couleur et le goût du dolo sont les trois principaux critères. Dans le Kouritenga, les premiers déterminants de la qualité sont la couleur, le goût et l'odeur du dolo. Dans le Kadiogo, le goût, le collant au toucher et le taux d'alcool apparaissent comme les principaux attributs.

Critères de choix des grains de sorgho pour la fabrication du dolo : La qualité du grain est le premier critère utilisé par $63 \%$ des brasseuses de dolo pour le choix des types de grains ou des variétés de sorgho. Elle est suivie du coût des grains et de l'appréciation de la qualité du dolo (goût plus ou moins doux, amer ou sucré) par les consommateurs locaux, respectivement cités par $19 \%$ et $12 \%$ des enquêtées contre $5 \%$ pour les autres motifs, tels que la disponibilité et les aspects culturels. Le test de Khi-2 montre qu'il existe une dépendance significative entre le type de grain de sorgho utilisé pour la préparation du dolo et la raison de son usage $(1-p=97,55 \%)$. En effet, il ressort des résultats qu'à l'exception du type de grain rouge léger et dur qui est utilisé autant pour sa qualité que parce qu'il est apprécié par les consommateurs, les autres types de grain sont surtout utilisés pour leur qualité (Figure 5). Dans chacune des trois provinces, le premier motif de choix de la variété est la qualité des grains, qui représente $55 \%$ des citations pour les brasseuses du Kadiogo, $59 \%$ pour le Mouhoun et $73 \%$ pour le Kouritenga.

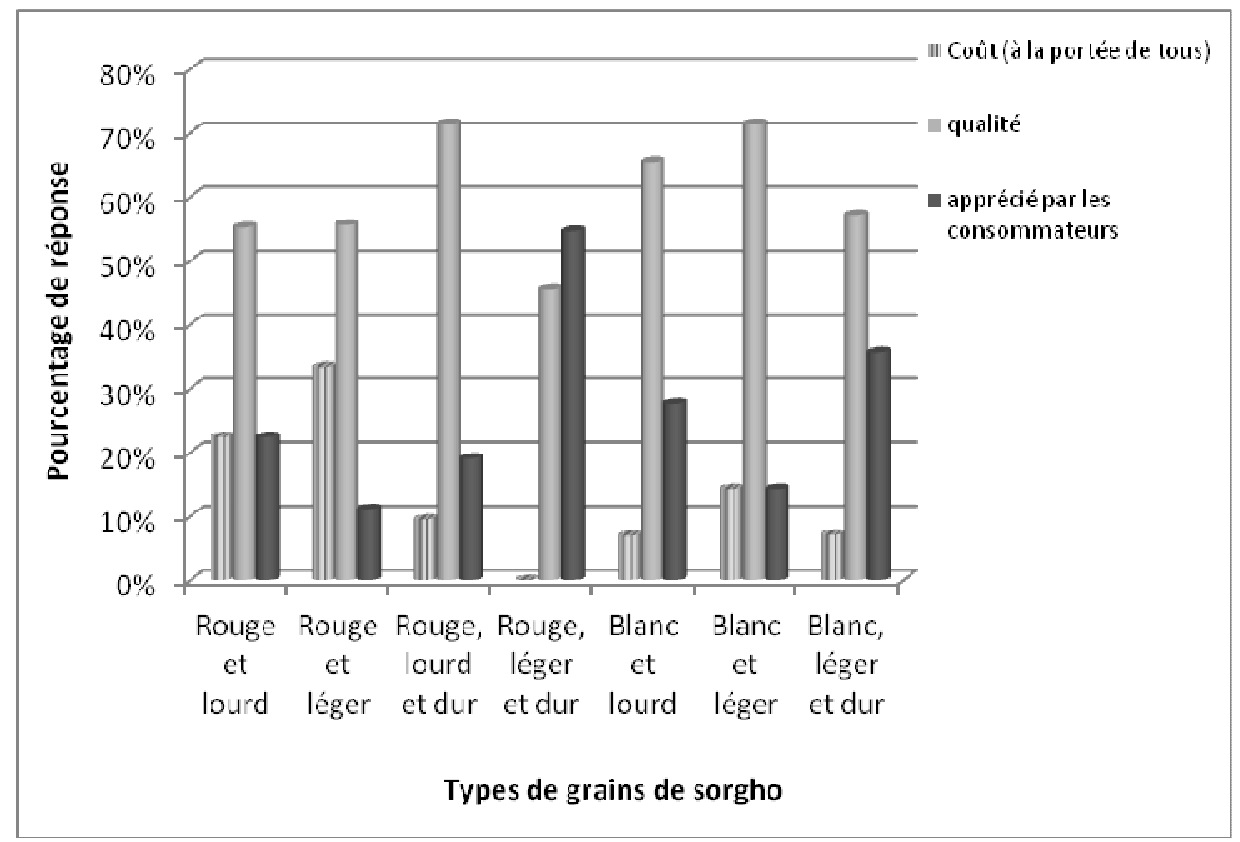

Figure 5 : Critères de choix des différents types de grains de sorgho pour la préparation du dolo Caractères de grain utilisés et préférés pour la fabrication d'un bon dolo

Les résultats de l'enquête montrent que les grains rouges et lourds et les grains rouges, lourds et durs sont les types de premiers choix pour respectivement $35 \%$ et $34 \%$ des brasseuses enquêtées (Figure $6 \mathrm{~A}$ ). Les grains rouges et légers sont un premier choix pour $18 \%$ des brasseuses et un deuxième choix pour $12 \%$ d'entre elles. Les grains blancs et lourds se placent en 4ème position des types de grains utilisés mais sont seulement choisis en 2ème choix. Cela signifie que ce type de grain constitue une solution de recours en cas de non ou faible disponibilité des trois premiers types de grains. Les résultats de l'enquête montrent également que la majorité des brasseuses, soit $80,3 \%$ estiment que les variétés de sorgho rouge donnent un bon dolo contre $19,7 \%$ pour le sorgho blanc. Pour l'ensemble des localités, les types de grain les plus aptes à donner un bon dolo selon l'appréciation des malteuses, sont par ordre d'importance les grains rouges et lourds $(29,9 \%)$, les grains rouges lourds et durs $(23,6 \%)$ et les grains rouges et légers $(17,3 \%)$, et les grains blancs et lourds (10,2\%) (Figure 6B). Le test de Khi-2 montre qu'il existe une relation très significative entre les provinces et les préférences sur les types de grains (1-p=99,99\%). En effet, les résultats 
indiquent que dans le Mouhoun les grains rouges lourds et durs et les grains rouges et lourds sont les types préférés mais les grains blancs et lourds sont le type de grain préféré par $16 \%$ des brasseuses. Dans le Kouritenga les grains rouges lourds et durs viennent largement en tête suivis par les grains rouges et lourds.
Dans la province du Kadiogo, les préférences des dolotières semblent moins bien établies que dans les deux autres provinces car elles sont essentiellement orientées vers les grains rouges et légers ou les grains rouges et lourds (Figure $6 \mathrm{C}$ ).

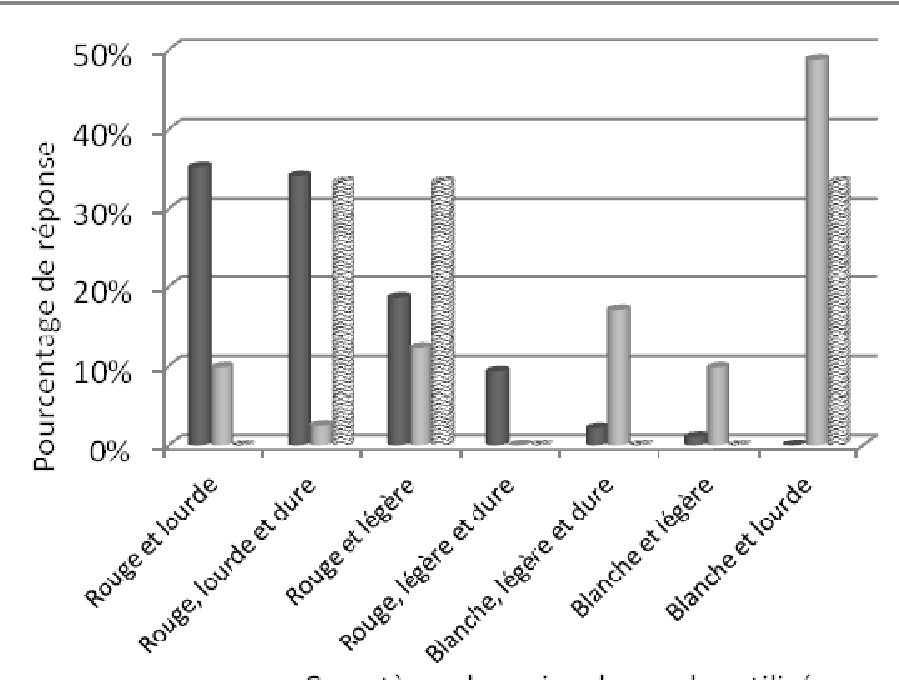

1er choix

- 2 ème choix

séme choix

Caractères de grains de sorgho utilisés

Figure 6A: Caractères de grain utilisés par les brasseuses pour la préparation du dolo pour l'ensemble des 10 localités étudiées

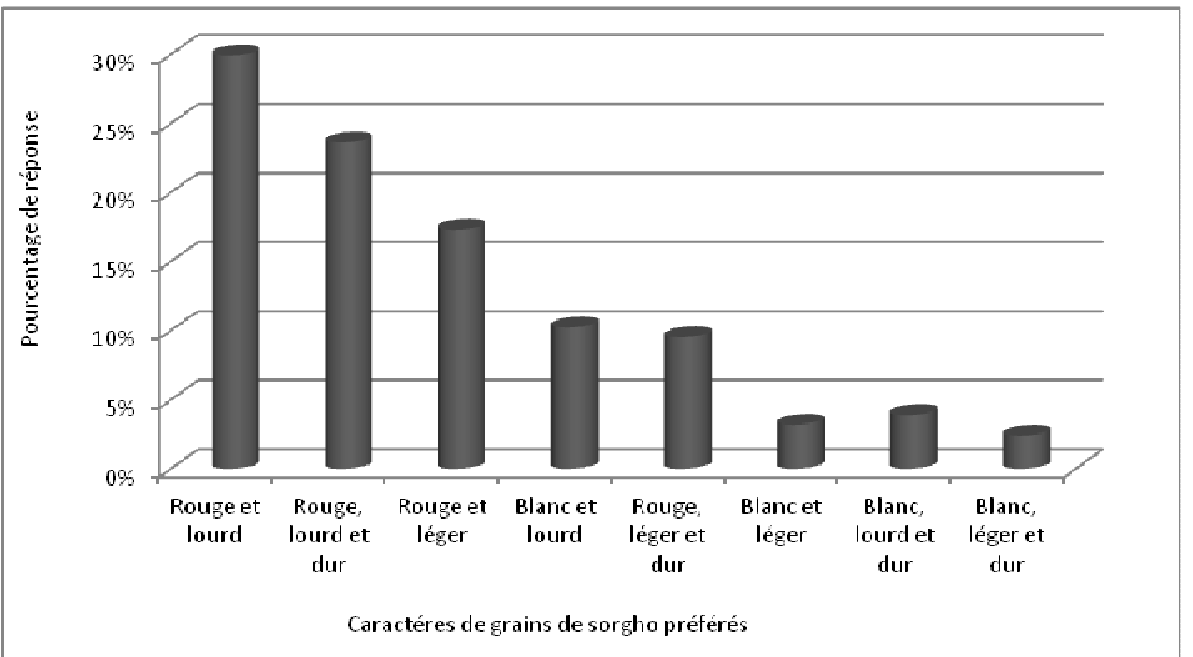

Figure 6B : Caractères de grain préférés par les brasseuses pour la préparation du dolo pour l'ensemble des 10 localités étudiées 


\section{Songre-Ouattara et al. J. Appl. Biosci. 2016 Quelles variétés de sorgho pour satisfaire les exigences}

des productrices de malt et de dolo au Burkina Faso?

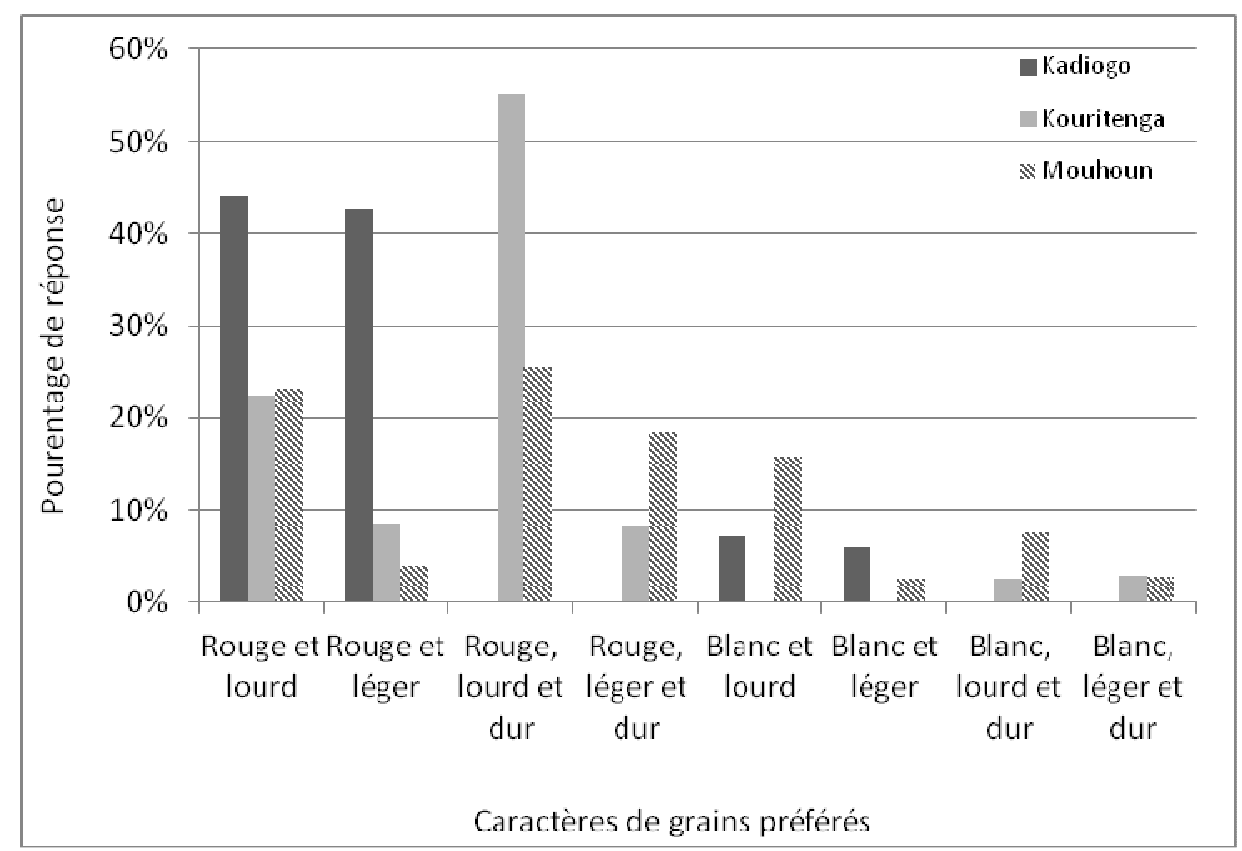

Figure 6C : Caractères de grain préférés par les brasseuses pour la préparation du dolo selon chaque province

\section{DISCUSSION}

Dans le secteur de la transformation des céréales, la qualité du grain est un critère déterminant car elle conditionne le type d'utilisation et l'aptitude à la transformation. Ainsi, notre étude s'est focalisée sur la détermination des types et caractéristiques des grains de sorgho préférés par les malteuses et les brasseuses de dolo pour produire un bon malt et/ou un dolo de qualité. Nos résultats montrent qu'un ensemble de caractères physiques, technologiques et organoleptiques du grain sont considérés par les malteuses et les brasseuses de dolo pour l'obtention de la qualité recherchée pour le produit transformé résultant de leur activité.

Caractéristiques de grains préférées pour l'obtention d'un malt de bonne qualité : L'étude confirme que le sorgho rouge est le type le plus utilisé par la majorité des malteuses dans les dix localités de notre étude comme déjà rapporté par Palé et al. (2010) pour une étude conduite dans quatre provinces du Centre et Centre-Est du Burkina Faso. Toutes les malteuses des dix localités étudiées indiquent leur préférence marquée pour les variétés de sorgho rouge. En effet, si l'utilisation du sorgho rouge est généralement justifiée par les malteuses par son aptitude à donner un dolo de couleur rouge à foncée et un goût bien apprécié des consommateurs, ses avantages reconnus pour la production du malt sont principalement la germination rapide des grains et la longue conservation du malt. La plus grande rapidité de germination attribuée au sorgho rouge est probablement lié au fait que la très grande majorité des variétés à grain rouge cultivées au Burkina ont des grains farineux avec une couche brune (Barro Kondombo et al., 2010) donc ayant des teneurs élevées en tanins, lesquels confèrent un avantage reconnu pour la vitesse de germination et la vigueur à la levée (Chantereau et al, 1983). Concernant l'appréciation d'un malt de sorgho de bonne qualité, les malteuses se rapportent au critère de taux élevé de germination et à des critères d'ordre organoleptique, principalement le goût sucré, l'odeur et la couleur du malt. Ces résultats convergent vers ceux obtenus par Palé et al. (2010). En effet, ces auteurs ont montré que les principaux critères utilisés par les acteurs de la filière dolo pour évaluer la bonne qualité d'un malt de sorgho sont le goût sucré, la présence ou l'absence de radicules (qui traduit le taux de germination des grains) et la couleur du malt. Ces observations plus ou moins empiriques des malteuses sur le fait que le taux de germination est un déterminant important de la qualité d'un malt destiné à la production du dolo sont corroborées par l'étude de Christian Konfo et al. (2014) qui ont souligné qu'un taux de germination élevé donne plus d'enzymes qui augmentent la production de sucre, soit des a et $\beta$ amylase qui dégradent l'amidon en mono et disaccharides (Hulse et al., 1980). De plus, 


\section{Songre-Ouattara et al. J. Appl. Biosci. 2016}

des productrices de malt et de dolo au Burkina Faso?

certains auteurs (Morrall et al., 1986; Lasekan et al., 1995) ont souligné que la germination des grains de sorgho pendant 3 à 7 jours à $30^{\circ} \mathrm{C}$ produit du malt avec un pouvoir diastasique élevé et des teneurs importantes en sucres solubles fermentescibles et en acides aminés libres. II est à noter que ce temps de germination correspond au temps moyen utilisé par les malteuses du Kadiogo selon les résultats de notre étude. L'étude révèle également que dans la quasitotalité des localités enquêtées, les caractéristiques de qualité de grain les plus désirées par les malteuses pour de nouvelles variétés de sorgho destinées à la production de malt sont: la bonne germination, la couleur rouge du grain et la dureté du grain. Les deux premiers caractères sont donc partagés entre les variétés déjà connues et utilisées et les variétés nouvelles souhaitées. La dureté du grain est jugée ou mesurée par les malteuses comme étant la résistance à l'écrasement lorsque le malt est légèrement broyé au moulin ou au pilon avant la 1ère cuisson. En fait ce caractère de dureté du grain constitue une exigence des brasseuses auprès des malteuses ou auprès $d u$ fournisseur de grains lorsque les brasseuses sont également malteuses. Les grains durs semblent conférer un avantage lors du brassage en facilitant la séparation après cuisson du mélange farine de malt et eau avec les sous-produits du brassage (drèche), ce qui permettrait d'éviter l'obtention d'un dolo pâteux qui n'est pas désiré. Les propriétés d'hydratation et ou de solubilité de l'amidon des grains pourraient aussi expliquer ces observations.

Caractéristiques de grains recherchés pour la fabrication du dolo et qualité du dolo : Pour environ 2/3 des brasseuses interviewées dans notre étude, la qualité du grain est le premier motif de choix d'une variété de sorgho pour la fabrication du dolo. Les déterminants de cette qualité du grain incluent principalement la dureté des grains et leur aptitude à bien germer. Pour ces deux attributs en particulier, les variétés de sorgho rouge présentent les types de grains les plus aptes à la fabrication du dolo. Parmi les variétés à grains rouges, celles à grains lourds (mâtures, bien remplis), et à grains lourds et durs sont les plus recherchées. Ces résultats sont en accord avec ceux de Palé et al (2010) qui ont montré que plusieurs caractéristiques physiques des grains de sorgho, notamment la taille, la dureté, la maturité, le poids sont rapportés par les malteuses comme des critères de choix car ils affectent la qualité du malt. Quant au sorgho blanc, cela signifie que ce type de grain constitue une solution de recours en cas de non ou faible disponibilité des trois premiers types de grains. Dans leur définition d'un bon dolo, les brasseuses enquêtées considèrent que les critères importants d'appréciation sont le goût, qui doit être sucré et peu aigre, la couleur rouge et une teneur en alcool assez élevée qui témoigne de la bonne fermentation. Des entretiens réalisés auprès des dolotières par Kaboré (2009) dans une autre localité du Burkina Faso (Kaya) indiquent que les consommateurs recherchent un dolo le plus naturel possible (sans addition d'alcool extérieur ou de molécules de synthèse), avec un goût agréable (ni acide, ni amer), sans odeur désagréable et qui ne rend pas les consommateurs malades. En outre, l'influence positive de la couleur rouge du grain sur la couleur du dolo a été rapportée par Odibo et al. (2002). Ces résultats corroborent également ceux de Kayodé et al. (2005) qui ont rapporté que les consommateurs africains préfèrent boire des bières traditionnelles colorées parce qu'ils pensent qu'elles sont meilleures pour la santé, lorsqu'elles sont naturelles sans ajout d'additifs. Cette connaissance empirique des consommateurs est de nos jours justifiée par la présence attestée de polyphénols dans ces bières traditionnelles, dont l'impact positif sur la santé a été démontré (Awika and Rooney, 2004). Même si cela n'a pas été mentionné explicitement, les brasseuses semblent aussi savoir que l'utilisation des grains de sorgho rouge permet de produire un dolo ayant une teneur élevée en alcool. Ceci a été vérifié par plusieurs études conduites au Burkina ayant montré que les sorghos rouges présentent une activité amylasique plus élevée que celle du sorgho blanc, du maïs et du mil (Traore et al., 2004). Dicko et al. (2006) et Agu et Palmer (1997) ont aussi montré que les variétés de sorgho à grains colorés ont des activités a et $\beta$-amylasiques plus élevées que celles des variétés à grains blancs. Les trois critères définissant la qualité du dolo présentent des niveaux d'importance un peu différents selon les provinces. Néanmoins, ces critères sont quasiment similaires à ceux rapportés par Palé et al. (2010) pour les 4 provinces considérées dans leur étude (Kadiogo, Ganzourgou, Kouritenga et Zoundwéogo). Selon ces mêmes acteurs, les critères majeurs de la qualité d'un bon dolo seraient le goût, la teneur en alcool et la bonne cuisson.

Comparaison des caractéristiques de grains recherchés par les malteuses et les brasseuses: Au regard des résultats obtenus sur les caractéristiques de grains recherchés par les malteuses et les brasseuses, il apparait que les variétés à grains rouge 


\section{Songre-Ouattara et al. J. Appl. Biosci. $2016 \quad$ Quelles variétés de sorgho pour satisfaire les exigences des productrices de malt et de dolo au Burkina Faso?}

sont mieux appréciées par ces deux groupes d'acteurs pour la production du malt et du dolo de sorgho en raison de leur bon pouvoir de germination et les meilleurs qualités organoleptiques qu'ils confèrent aux produits élaborés. Au sein des variétés de sorgho rouge, les caractères grains lourds, ou grains lourds et durs sont les plus recherchés par les brasseuses des trois provinces probablement parce que les grains lourds sont des grains matures et bien remplis, donc

\section{CONCLUSION}

Notre étude souligne les perceptions de la qualité d'un grain de sorgho pour un bon malt et un bon dolo par les malteuses et brasseuses de dolo des trois provinces du Burkina Faso. Selon les résultats obtenus, les variétés de sorgho rouge, comparativement au sorgho blanc,

\section{REMERCIEMENTS}

Ces travaux ont été réalisés dans le cadre du grant « Updating knowldege on demands for grain and stalk quality of sorghum varieties in Burkina Faso » financé par le Collaborative Research program Dryland Cereals coordonné par l'ICRISAT. Nous tenons à remercier MM Abdoulaye Ouédraogo de l'AMSP et Adama Sidibé de I'UGCPA-BM pour leur appui dans la réalisation des

\section{REFERENCES}

Agu RC, Palmer GH, 1997. Effect of mashing procedures on some sorghum varieties germinated at different temperatures. Process Biochemistry 32(2):147-158, doi:10.1016/ S0032-9592(96)00074-X.

Awika JM, Rooney LW, 2004. Sorghum phytochemicals and their potential impact on human health. Phytochemistry 65(9):1199-1221. doi.org/ 10.1016/j.phytochem.2004.04.001.

Barro-Kondombo C, Sagnard F, Chantereau J, Deu M, vom Brocke K, Durand P, Goze E, Zongo J-D, 2010. Genetic structure among sorghum landraces as revealed by morphological variation and microsatellite markers in three agroclimatic regions of Burkina Faso. Theoretical Applied Genetics 120:1511-1523.

Belliard F, 2001. La préparation de bière de sorgho chez les joôhé (Burkina Faso): étude ethnolinguistique d'une technique. In : Journal des africanistes, 2001, tome 71, fascicule 2. pp. 49-76.

Broutin C, Totté A, Tine E, François M, Carlier R, Badini Z, 2003. Transformer les céréales pour les nouveaux marchés urbains : opportunités aptes à donner une plus grande quantité de malt de bonne qualité. Cependant, la dureté pourrait traduire à la fois un grain sec capable d'absorber la quantité d'eau nécessaire pour une bonne germination et pour lequel le malt obtenu s'écrase difficilement pour ne pas donner des farines de granulométrie fine qui semblent affecter l'empâtage dont le but est d'extraire les enzymes et les sucres fermentescibles du malt.

sont les utilisées et préférées pour l'activité de maltage et de production de dolo. Cependant, au sein des variétés à grain rouge, les caractères de grains plus aptes à donner un bon malt et un bon dolo préférés sont ceux de type lourds, ou lourds et durs.

enquêtes de terrain respectivement dans les provinces du Kouritenga et du Mouhoun Sandrine Dury pour son appui à l'élaboration des questionnaires et l'exploitation des données obtenues. Nous remercions également les enquêteurs et l'ensemble des producteurs, malteuses et brasseuses de dolo interviewées dans les trois provinces.

pour des petites entreprises en Afrique. GRET, Paris : 296p.

Chantereau J, 1983. La sélection de lignées de sorgho vigoureuses à la levée pour la zone Centre Nord du Sénégal. In : Réunion des Comités Scientifiques du Projet Régional d'Amélioration des Mil, Sorgho, Niébé et Maïs. Sénégal : GERDAT-IRAT, 4 p.

Chevassus-Agnes S, Favier JC, Joseph A, 1979. Traditional technology and nutritive value of Cameroon sorghum beers. Cahiers de l'Onarest 2:83-112.

Christian Konfo TR, Euloge Adjou S, DahouenonAhoussi E, Mohamed Soumanou M, Dominique Sohounhloue CK, 2014. Physicochemical profile of malt produced from two sorghum varieties used for local beer (Tchakpalo) production in Benin. International Journal of Biosciences 5(1):217-225, doi.org/10.12692/ijb/5.1.217-225.

Dicko MH, Gruppen H, Traoré AS, Voragen AGJ, van Berkel WJH, 2006. Sorghum grain as human food in Africa : relevance of content of starch 


\section{Songre-Ouattara et al. J. Appl. Biosci. 2016 Quelles variétés de sorgho pour satisfaire les exigences}

des productrices de malt et de dolo au Burkina Faso?

and amylase activities. African Journal of Biotechnology 5: 384-395.

DSA-BF, 2001. Production du Sorgho (Sorghum production). MAHRH, DGPSA, DSA, BF.

Haggblade S, Holzapfel H, 2004. Industrialization of Africa's indigenous beer brewing. In: Streinrous K.H. Industrialization of indigenous fermented foods. 2nd ed. New York, USA: CRC Press.

Hounhouigan J, Fliedel G, 2010. Production de malt de sorgho ou de mil de qualité pour la production alimentaire artisanale ou semi-industrielle en Afrique de l'Ouest. In « le Projet DURAS » : des partenariats innovants pour le développement durables. Extrait du dossier thématique d'Agropolis International : 41-43.

Hulse JH, Laing M E, Pearson EO, 1980. Sorghum and the millets: their composition and nutritive value. Academic press. London. $997 \mathrm{p}$.

Kayode PAP, Adegbidi A, Hounhouigan DJ, Linnermann RA, Nout MJR, 2005. Quality of farmers' varieties of sorghum and derived foods as perceived by consumers in Benin. Ecology of Food and Nutrition 44:271-294, doi: 10.1080/03670241003695773.

Lasekan OO, Idowu MA, Lasekan W, 1995. Effect of germination and degree of grind (coarse/fine) on the extract and sugar content of sorghum malts. Food Chemistry 53(2):125-128, doi:10.1016/0308-8146(95)90776-4.

Lyumugabe F, Gros J, Nzungize J, Bajyana E, Thonart $P, 2012$. Characteristics of African traditional beers brewed with sorghum malt: a review. Biotechnologie, Agronomie, Société et Environnement 16 (4):509-530.

Maha FE, Runqiang Y, Qianghui G, \& Zhenxin G, 2014. Major nutrient compositions and functional properties of sorghum flour at $0-3$ days of grain germination. International Journal of Food Sciences and Nutrition 65(1):48-52, doi:10.3109/09637486.2013.836736.

Malomo Olu, Alamu AE, Oluwajoba SO, 2013. Effect of Processing on Total Amino Acid Profile of Maize and Cowpea Grains. Journal of Advanced Laboratory Research in Biology $4(2): 77-82$.

Ministère de l'Agriculture, des Ressources Hydrauliques de l'Assainissement et de la Sécurité Alimentaire-Direction Générale des Études et Statistiques Sectorielles (MARHASA/DGESS), 2015. Résultats définitifs de la champagne agricole et de la situation alimentaire et nutritionnelle, Ouagadougou, Burkina Faso.

Morrall P, Boyd HK, Taylor JRN, Van Der Walt WH, 1986. Effect of germination time, temperature and moisture on malting of sorghum (Sorghum bicolor). Journal of the Institute of Brewing 92 (5):439-445, doi: 10.1002/j.2050-0416.1986 .tb04437.x.

Odibo FJC, Nwankwo LN, Agu RC (2002). Production of malt extract and beer from Nigerian sorghum varieties. Process Biochemistry 37: 851-855.

Palé S, Taonda SJB, Bougouma B, Masson S, 2010. Sorghum malt and traditional beer (dolo) quality assessment in Burkina Faso. Ecology of Food and Nutrition 49(2):129-141, doi: 10.1080/03670241003697573.

Rai KN, Gowda CLL, Reddy BVS, Sehgal S, 2008. Adaptation and Potential Uses of Sorghum and Pearl Millet in Alternative and Health Foods. Comprehensive Reviews in Food Science and Food Safety 7 (4):320-396, doi: 10.1111/j.1541-4337.2008.00049.x.

Saleh ASM, Zhang Q, Chen J, Shen Q, 2013. Millet Grains : Nutritional Quality, Processing, and Potential Health Benefits. Comprehensive Reviews in Food Science and Food Safety 12(3): 281-295, doi: 10.1111/1541-4337. 12012.

Songré-Ouattara LT, Bationo F, Parkouda C, Dao A, Bassole IHN, Diawara B, 2015. Qualité des grains et aptitude à la transformation : cas des variétés de Sorghum bicolor, Pennisetum glaucum et Zea mays en usage en Afrique de I'Ouest. International Journal of Biological and Chemical Sciences 9 (6): 2819-2832, doi:10.4314/ijbcs.v9i6.23.

Thaoge ML, Adams MR, Sibara MM, Watson TG, Taylor JRN and Goyvaerts EM, 2003. Production of improved infant porridges from pearl millet using a lactic acid fermentation step and addition of sorghum malt to reduce viscosity of porridges with high protein, energy and solids (30\%) content. Journal of Microbiology and Biotechnology $19: 305-310$, doi: 10:1023614526667.

Traore T, Mouquet C, Icard-Vernière C, Traore AS, Treche S, 2004. Changes in nutrient composition, phytate and cyanide contents and $a$-amylase activity during cereal malting in 
small production units in Ouagadougou (Burkina Faso). Food Chemistry 88 (1):105114, doi:10.1016/j.foodchem.2004.01.032.

Taylor JRN, Dewar J, 2001. Development in sorghum food technologies. Advances in Food and Nutrition Research 43, 217-264, doi : 10.1016/S1043-4526(01)43006-3.

Tchuenbou FL, 2006. Maltage du mil et du sorgho. Mise au point d'outils et méthodes et aptitude au maltage de quelques variétés du Burkina Faso et du Bénin. Thèse de Master of Science, Université de Montpelier 2.

Wenzel WG, Pretorius AJ, 1994. The Relationship between Characteristics Associated with Malt Quality of Grain Sorghum. Angewandte Botanik 68(5-6):143-145. 